\title{
UPB at SemEval-2021 Task 7: Adversarial Multi-Task Learning for Detecting and Rating Humor and Offense
}

\author{
Răzvan-Alexandru Smădu, Dumitru-Clementin Cercel, Mihai Dascalu \\ University Politehnica of Bucharest, Faculty of Automatic Control and Computers \\ razvan. smaduastud. acs . upb.ro \\ \{dumitru.cercel, mihai.dascalu\}@upb.ro
}

\begin{abstract}
Detecting humor is a challenging task since words might share multiple valences and, depending on the context, the same words can be even used in offensive expressions. Neural network architectures based on Transformer obtain state-of-the-art results on several Natural Language Processing tasks, especially text classification. Adversarial learning, combined with other techniques such as multi-task learning, aids neural models learn the intrinsic properties of data. In this work, we describe our adversarial multi-task network, AMTLHumor, used to detect and rate humor and offensive texts from Task 7 at SemEval-2021. Each branch from the model is focused on solving a related task, and consists of a BiLSTM layer followed by Capsule layers, on top of BERTweet used for generating contextualized embeddings. Our best model consists of an ensemble of all tested configurations, and achieves a $95.66 \%$ F1-score and $94.70 \%$ accuracy for Task 1a, while obtaining RMSE scores of 0.6200 and 0.5318 for Tasks $1 \mathrm{~b}$ and 2 , respectively.
\end{abstract}

\section{Introduction}

Sentiment analysis studies expressed opinions and feelings, affective states, and subjective information introduced while conveying ideas to others. In recent years, social media has encountered a major change in ways of interaction and in the freedom of expressing moods to others. For example, individuals frequently use emojis, or even abbreviations, to emphasize their feelings. Therefore, new machine learning systems are developed to predict sentiment valences; however, this task is not trivial, as the detection of sentiments is challenging even for humans. This is mainly generated by the diversity of contexts and differences among people, such as socio-cultural status, age, or gender. For example, certain jokes might be confusing due to past experiences or the current mood while reading the joke. Similarly, language ambiguity can introduce difficulties in understanding the text and in altering the perceived emotions.

Moreover, individuals express a wide range of sentiments, such as happiness, humor, or anger. Words that are used to express such feelings, might share multiple valences and meanings, depending on the context. Therefore, a sequence of words that is, for example, humorous in a certain context, can be considered offensive in another situation. Such examples can rely on bad jokes regarding a person's position in society, ethnicity, or political ideology. Another characteristic that impacts a text's level of humor is how clear the idea is conveyed to the reader.

In this regard, SemEval-2021 Task 7 - HaHackathon: Detecting and Rating Humor and Offense (Meaney et al., 2021) introduced four subtasks: Task $1 a$ (classification) includes the class of the text, which can be either humorous or not; Task $1 b$ (regression) presents the level of humor as a value between 0 and 5; Task $1 c$ (classification) covers the rating of the class of being controversial (i.e., some annotators considered a text to be humorous, while others considered the opposite); Task 2 (regression) contains the level of offensiveness from the text (i.e., how offensive is the text as an aggregate score agreed by all annotators).

Each example in the dataset contains four labels, one for each task. The labels for Tasks $1 \mathrm{~b}$ and $1 \mathrm{c}$ depend on whether the text contains humor or not. Task 2 is independent of Task 1 and all examples from the dataset have assigned scores corresponding to their level of offensiveness.

In this work, we introduce an adversarial multitask learning (Liu et al., 2017) architecture to detect humor in texts, as well as other related sub-tasks. We focus on Tasks 1a, 1b, and 2, while using the data for all tasks during training. Our model con- 
siders BERTweet (Nguyen et al., 2020) for contextualized embeddings, followed by Long ShortTerm Memory (LSTM) (Hochreiter and Schmidhuber, 1997) and Capsule layers (Sabour et al., 2017) as feature extractors. We observe that adversarial learning increases performance for certain choices of hyper-parameters. Moreover, training the branch for Task 1c on the data for Task 1a improves the performance for Task 1a. In addition, an ensemble method increases the overall performance on all tasks.

The paper is organized as follows. Section 2 presents related work associated with the provided tasks. Section 3 describes our method, followed by details on the experimental setups and results. Finally, Section 5 provides conclusions and future research paths.

\section{Related Work}

Several studies (Roberts et al., 2012; Marasović and Frank, 2018; Zaharia et al., 2020) address the problem of detecting sentiments from texts by using multiple related tasks to further improve the performance of the model. Adversarial multi-task learning was introduced by Liu et al. (2017) and consists of using multiple branches for each individual task (i.e., private branches), and another branch shared among all tasks (i.e., shared branch). A discriminator trained to distinguish between features from multiple tasks is used to separate shared and task-related features in the latent representation from each branch. This is the Adversarial Shared-Private Multi-Task Learning (ASP-MTL) framework that was also employed in other works such as (Marasović and Frank, 2018), where the goal was to label opinions and the associated semantics from texts. Results showed that ASP-MTL might not achieve better results when compared to classical MTL, and there are other factors that should be taken into consideration, such as dataset split and hyper-parameters.

Other works (Zhou et al., 2019; Spiliopoulou et al., 2020) used the multi-task technique alongside adversarial learning, but in other configurations than ASP-MTL. For example, Zhou et al. (2019) employed a model that has a shared feature extractor, and then it is followed by branches for each task with an attention mechanism for the first layer from each branch. By adding new branches to the model, as well as adversarial learning, the F1-score increased when compared to the baseline model. Spiliopoulou et al. (2020) adopted domain adaptation to improve the performance of a classifier. Their idea was to add a discriminator after the feature extractor, as a separate branch, that is used to distinguish between different domains. The experiments showed that adversarial learning aided in reducing the bias found in the dataset, thus increasing the model's capability to generalize.

There are multiple Natural Language Processing (NLP) techniques that can be effectively employed to perform text classification in general, as well as sentiment analysis as a specific task (Paraschiv and Cercel, 2019; Tanase et al., 2020b,a; Paraschiv et al., 2020). For example, neural network methods can be used to detect propaganda in articles by pretraining models on related tasks (Vlad et al., 2019) or emotions in memes using multimodal multi-task learning (Vlad et al., 2020a,b). Other methods rely on classical machine learning methods (e.g., Support Vector Machine, Naive Bayes, or Random Forest classifiers) and can be successfully used for related tasks, such as fake news detection, obtaining accuracies over $90 \%$ on specific datasets (Dumitru and Rebedea, 2019; Busioc et al., 2020).

\section{Method}

\subsection{Corpus}

The provided dataset for SemEval-2021 Task 7 (Meaney et al., 2021) consists of 10,000 texts (6,179 texts are considered to have humor, while the rest do not present humor), written in English, with different degrees of humor, and labeled by different categories of people; therefore the labeling is highly subjective. Given the texts with humor, the scores for the Task $1 \mathrm{~b}$ follow a Gaussian distribution, with the mean of 2.24 (out of 5) and the standard deviation of 0.56 . Also, approximately $46.39 \%$ of the texts for Task 1c were labeled as controversial. Furthermore, $42.46 \%$ of the texts were rated as not being offensive at all for the last task, while the 75th percentile is 1.55 (out of 5) for the offensive samples. This means that the data is biased towards not being offensive for the Task 2 .

The dataset was already split into train/dev/test sets, such that 8,000 samples were used for training, 1,000 for development, and 1,000 for testing, following similar distributions. Only the entries from training and development sets were provided with annotations during the competition. The annotations for the test set were provided after the evaluation phase ended. 


\subsection{Neural Architecture}

An increasing trend of applying adversarial learning alongside MTL leads to improvements in baseline models (Liu et al., 2017; Marasović and Frank, 2018; Srivastava et al., 2020). The underlying intuition for this setting is to concurrently use potential information hidden in the correlation between multiple tasks through MTL, while storing this representation in a shared-private architecture.

Inspired by the three previously mentioned works, Figure 1 shows an overview of our proposed adversarial multi-task learning architecture, namely AMTL-Humor. Blue denotes the shared branch among all tasks, whereas task-specific branches are in white. The discriminative part from the network is represented in green.

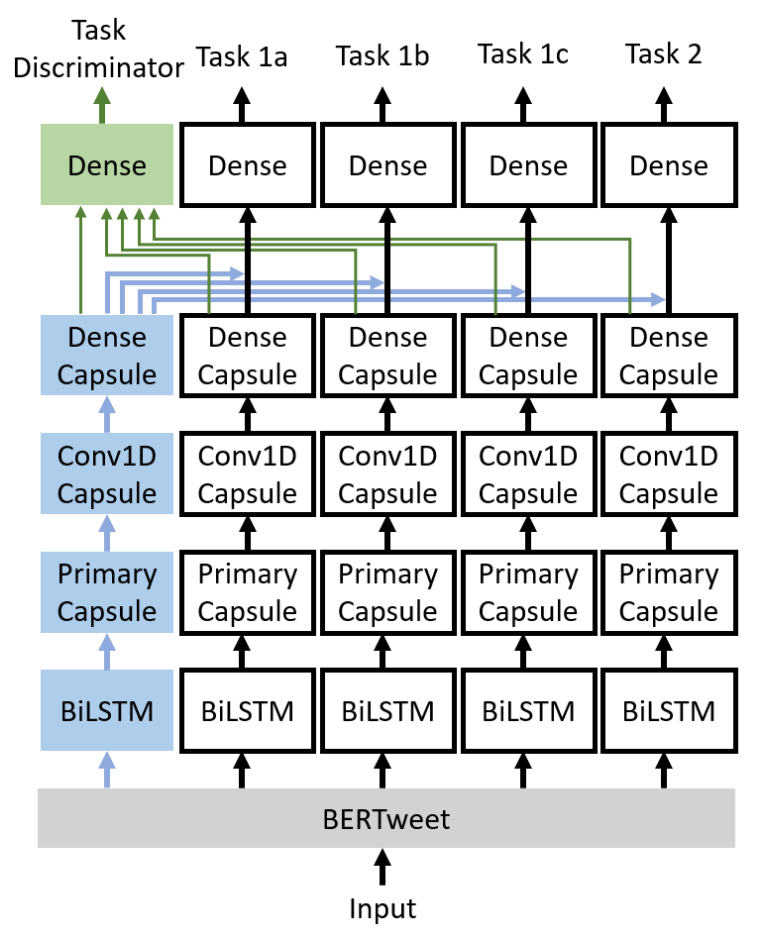

Figure 1: Overview of the AMTL-Humor architecture.

We employ a pre-trained Transformer-based model (Vaswani et al., 2017) for generating contextualized embeddings, namely BERTweet, which was trained on $850 \mathrm{M}$ tweets written in English. The input is preprocessed in the same way as the input on which the Transformer was trained on, by using the TweetTokenizer from NLTK toolkit (Bird et al., 2009). The representation of the input text given by the BERTweet is further fed into five branches. One branch is shared among all other tasks and learns information that is non-task-specific. The output of this branch is concatenated with each private branch, which is then fed to a linear classifier responsible for outputting the final results. A discriminator classifier takes as input the outputs from all branches before the concatenation, having as goal to learn which representation is from what task. In this scenario, labels concerning the branch from which the discriminator took the values are known.

Each branch is composed of five layers, based on a Recurrent Neural Network (Cho et al., 2014) and Capsule layers (Lei et al., 2019; Srivastava et al., 2020). First, the Bidirectional LSTM (BiLSTM) layer acts as a feature extractor and encodes the high-level features. It is used to provide a latent representation of the input space that captures the dependencies from both left-to-right and right-toleft. Second, the Primary Capsule layer captures the local order of words in the latent space of the BiLSTM output. It encodes the hidden properties, such as positional information for words and the relations between words, from the latent representation into a vector. Third, the Convolutional $1 D$ Capsule layer learns the child-parent relationships and predicts the parent Capsules in the next layer by using a dynamic routing algorithm (Sabour et al., 2017). Fourth, the Dense (Fully Connected) Capsule layer takes the output of the previous layer, flattens it into a list of Capsules, and then a Capsule is produced to encode the features and their probability by using routing-by-agreement. Fifth, the Dense (Fully Connected) layer is the classical Perceptron that takes as input the representation from the last Capsule layer and computes the final output for that branch (which can be for either classification or regression task).

The idea of using Capsule layers was first introduced in Computer Vision by Sabour et al. (2017) and was studied in various NLP tasks (Yang et al., 2018; Xiao et al., 2018; Wang, 2019; Lei et al., 2019; Srivastava et al., 2020; Zaharia et al., 2020). Capsule networks are based on how human vision works - i.e., Capsules create groups of neurons that are specialized in recognizing certain properties of the input (Sabour et al., 2017).

\subsection{Optimization Problem}

Binary cross-entropy loss is used for the classification problems (i.e., humor classification and humor controversy tasks), while the mean squared error loss function is used for regression tasks (i.e., tasks for predicting humor and offensive scores). Thus, 
the loss associated with the tasks is computed as follows:

$$
L_{\text {tasks }}=\sum_{i=1}^{4} \alpha_{i} L_{i}
$$

where $\alpha_{i}$ represents the weight associated with the task $i$ and $L_{i}$ is the loss value for the task $i$.

A problem arises when employing standard MTL, namely, there is a high possibility that part of the shared information may arrive inside the taskrelated space and vice-versa. This problem can be alleviated by using adversarial learning to enforce the network to make the separation between the private and shared representations. Also, a generalization of the loss function (Liu et al., 2017) for training the Generative Adversarial Networks (Goodfellow et al., 2014) is used because we are dealing with multiple tasks, thus multiple classes for the discriminator:

$L_{A d v}=\min _{\theta_{S}}\left(\lambda \max _{\theta_{D}}\left(\sum_{k=1}^{5} \sum_{i=1}^{N_{k}} d_{i}^{k} \log \left[D\left(E\left(x^{k}\right)\right)\right)\right]\right)$

In order to further ensure the separation between the shared and private branches, we add an orthogonality constraint function (Bousmalis et al., 2016) to the objective function during the training, which is defined as:

$$
L_{\text {Diff }}=\sum_{k=1}^{4}\left\|S^{k^{\top}} H^{k}\right\|_{F}^{2}
$$

where $S^{k}$ and $H^{k}$ are the outputs of the from each branch, before the final classification layer, and $\|\cdot\|_{F}$ is the Frobenius norm.

The total loss for the optimization problem is the sum of all three losses, parametrized by $\lambda$ and $\gamma$, which control the importance for the adversarial and orthogonality losses, respectively:

$$
L_{\text {Total }}=L_{\text {Tasks }}+\lambda L_{A d v}+\gamma L_{D i f f}
$$

\subsection{Experimental Settings}

\subsubsection{Text Preprocessing}

The texts were not cleared; as such emojis were transformed into text to be further considered as tokens. The TweetTokenizer applies a normalization step to change noise constructs from the text (e.g., URLs) into special tokens. Missing values were replaced with 0 instead of $\mathrm{NaN}$ for Tasks $1 \mathrm{~b}$ and $1 \mathrm{c}$ that depend on whether the text was considered to be humorous or not in Task 1a.

\subsubsection{Implementation Details}

A public implementation of the Capsule layer ${ }^{1}$ was converted from TensorFlow 1.x to TensorFlow 2.4; this updated version was also publicly ${ }^{2}$. Also, the gradient reversal layer (Ganin and Lempitsky, 2015) was used for implementing the adversarial learning between the discriminator and the shared branch, which negates the gradient for the adversarial loss during back-propagation.

The Adam optimizer (Kingma and Ba, 2014) with a learning rate of 0.001 was considered for training the networks between 10 and 20 epochs, while the batch size was set between 8 and 16 . The size of the hidden state for BiLSTMs was set to 128 , with a dropout rate of 0.5 , as suggested by Srivastava et al. (2014). The number of filters in the Primary Capsule layer was set to 8 and the size of the kernel was set to $3 \times 1$. Also, the number of filters in the Convolutional 1D Capsule layer was set to 4 and the size of the kernel was set to $3 \times 1$. Moreover, the output size was set to $4 \mathrm{x} 1$ for all used Capsules, running the routing-by-agreement algorithm for 3 iterations.

During the experiments, different AMTL-Humor configurations were considered by enabling or disabling adversarial learning, the orthogonality constraints, and different parts of the architecture. Table 1 introduces our configurations, with values lower than 0.5 for hyper-parameters $\lambda$ and $\gamma$, and with conditions concerning adversarial loss being prioritized over the orthogonality loss.

\subsubsection{Baseline Models}

Two configurations were used as baseline, namely: a) a larger network (i.e., MTL-Large) with 512 hidden states for LSTM cells, 16 primary Capsule filters, and 8 filters for the convolutional Capsule layer with $5 \times 1$ filters, and b) a smaller model (i.e., $M T L-S m a l l)$ with the previously introduced parameters, sharing the same configurations as the other networks we have tested that use adversarial learning. The optimization step does not involve adversarial learning, nor the orthogonality constraints.

\subsubsection{AMTL-Humor Variants}

Different models based on the ASP-MTL architecture were tested on the provided dataset. Our base model AMTL-Humor has two variants (i.e. AMTL-

\footnotetext{
${ }^{1}$ https: / /github.com/naturomics / CapsLayer

${ }^{2}$ https://github.com/razvanalex/ CapsLayer
} 


\begin{tabular}{lccccc}
\hline Model & $\begin{array}{c}\text { Adversarial } \\
\text { Training }\end{array}$ & $\begin{array}{l}\text { Orthogonality } \\
\text { Constraints }\end{array}$ & $\begin{array}{c}\text { Capsule } \\
\text { Layers }\end{array}$ & $\lambda$ & $\gamma$ \\
\hline MTL-Large & - & - & - & 0 & 0 \\
MTL-Small & - & - & - & 0 & 0 \\
AMTL-LSTM & $\checkmark$ & $\checkmark$ & - & 0.1 & 0.01 \\
AMTL-Adv & $\checkmark$ & - & $\checkmark$ & 0.1 & 0 \\
AMTL-Humor-1 & $\checkmark$ & $\checkmark$ & $\checkmark$ & 0.1 & 0.01 \\
AMTL-Humor-2 & $\checkmark$ & $\checkmark$ & $\checkmark$ & 0.5 & 0.1 \\
AMTL-T1a-Twice* & $\checkmark$ & $\checkmark$ & $\checkmark$ & 0.05 & 0.01 \\
\hline
\end{tabular}

Table 1: The settings for each configuration. The star $(*)$ indicates that the model uses the third branch (i.e., for Task 1c) to solve Task 1a instead. Note that two branches are used for solving Task 1a.

Humor-1 and AMTL-Humor-2), as presented in Table 1 . The only difference between these is the choice for the $\lambda$ and $\gamma$ parameters. Also, AMTL$A d v$ is a model without orthogonality constraints, whereas AMTL-LSTM does not contain the Capsule layers in the architecture. Throughout our experiments, we observed that the network always outputs 0 on Task $1 \mathrm{c}$ for all inputs; therefore, the model does not manage to learn anything. As such, the AMTL-Tla-Twice configuration uses the Task 1a branch twice for both the first and third branches.

\subsubsection{Ensembles}

Ensemble learning was also considered; the modal value (i.e., the most frequent class) is taken for classification tasks, while the average of scores is used for regression tasks. Ensemble- 1 combines the results obtained by MTL-Large, AMTL-Humor-1, AMTL-T1a-Twice, and AMTL-Adv respectively, while Ensemble-2 adds to the previous list the models MTL-Small, AMTL-LSTM, and AMTLHumor-2.

\subsubsection{Evaluation Metrics}

F1-score and accuracy were used to evaluate the classification tasks, whereas root mean squared error (RMSE) was used to assess performance on the regression tasks from the SemEval-2021 Task 7 competition.

\section{Results}

Table 2 presents the results obtained on both development and test sets. On the development set, we observe that increasing the values of the parameters impacts more the regression tasks (i.e., higher RMSE values). Using only adversarial learning, without orthogonality constraints seems to add a small improvement in our case. Removing the ad- versarial learning has a very small negative impact on Task 1a, and a decrease of RMSE for the regression tasks. Removing only the Capsule layers seems to generate an improvement for this development set. The AMTL-T1a-Twice configuration achieves the highest F1-score due to the symmetry of our architecture. The increase in performance is notable, more than $2 \%$ when compared to AMTLHumor-1.

We observe that our baseline models achieve on the test set similar scores for Task 1a, whereas differences exist for the other two tasks, with no clear better configuration. The larger network tends to learn better on Task $1 \mathrm{~b}$, whereas RMSE is worse on Task 2 when compared to MTL-Small. All models that use our adversarial multi-task framework show a small improvement over the baselines on Task 2; nevertheless, almost all models perform worse on Task $1 \mathrm{~b}$, especially our system (i.e., AMTLHumor-1) that registers the highest RMSE scores. All adversarial models achieve or the Task 1a similar results, or even better when compared to the baseline models, and this was our main focus during the training.

The AMTL-T1a-Twice model manages to obtain the highest F1-score on one of the two branches for Task 1a. However, accuracy and F1 scores were low when making predictions for Task 1.c ( $46.34 \%$ and $61.18 \%$, respectively). These low performance values may be indicative of bias in the dataset towards either 0 or missing values for this task.

Ensemble models complement the inner configurations and provide more stable predictions. Ensemble-1 was the model submitted during the competition for evaluation. The differences between our Ensemble-1 configuration and the best models reported for each task were the following: 


\begin{tabular}{lcccccccc}
\hline \multirow{2}{*}{ Model } & \multicolumn{3}{c}{ Development Set } & \multicolumn{4}{c}{ Test Set } \\
& \multicolumn{2}{c}{ Task 1a } & Task 1b & Task 2 & \multicolumn{2}{c}{ Task 1a } & Task 1b & Task 2 \\
& F1 (\%) & Acc. (\%) & RMSE & RMSE & F1 $(\%)$ & Acc. (\%) & RMSE & RMSE \\
\hline MTL-Large* & 94.80 & $\mathbf{9 3 . 4 0}$ & 0.6434 & 0.7390 & 95.05 & 93.90 & 0.6359 & 0.5891 \\
MTL-Small & 93.08 & 90.90 & 0.6747 & 0.6649 & 95.07 & 93.80 & 0.6699 & 0.5796 \\
AMTL-LSTM & 94.12 & 92.50 & 0.6939 & 0.6738 & 95.39 & 94.30 & 0.6569 & 0.5552 \\
AMTL-Adv* & 93.52 & 92.10 & 0.6979 & 0.7053 & 93.61 & 92.40 & 0.6882 & 0.5631 \\
AMTL-Humor-1* & 93.27 & 91.40 & 0.7294 & 0.7294 & 94.93 & 93.80 & 0.7116 & 0.5616 \\
AMTL-Humor-2 & 93.75 & 92.10 & 0.7426 & 0.6759 & 95.32 & 94.30 & 0.7151 & 0.5680 \\
AMTL-T1a-Twice & $\mathbf{9 5 . 8 8}$ & 92.09 & 0.6977 & 0.6881 & $\mathbf{9 6 . 2 9}$ & 92.85 & 0.6774 & 0.5772 \\
Ensemble-1* & 94.05 & 92.50 & $\mathbf{0 . 6 3 6 1}$ & 0.6656 & 95.66 & 94.70 & 0.6200 & 0.5318 \\
Ensemble-2 & 94.54 & 93.10 & 0.6383 & $\mathbf{0 . 6 4 9 7}$ & 95.82 & $\mathbf{9 4 . 9 0}$ & $\mathbf{0 . 6 1 6 4}$ & $\mathbf{0 . 5 2 7 0}$ \\
\hline
\end{tabular}

Table 2: The results obtained on both development set (left) and test set (right). The best scores are marked in bold. The star $\left(^{*}\right)$ indicates the models we used for the submissions during the evaluation phase.

$2.88 \%$ less F1-score and 3.5\% less accuracy for Task 1a, 0.124 higher RMSE for Task 1b, and 0.119 higher RMSE for Task 2. During the postevaluation phase, we assessed the Ensemble-2 configuration that performs better than Ensemble- 1 on all tasks by a small margin (i.e., less than $1 \%$ ).

\subsection{Visualizations}

t-SNE visualizations (Van der Maaten and Hinton, 2008) were considered to better grasp the adequacy of our model. t-SNE is an algorithm used to visualize high-dimensional data into a two-dimensional representation by minimizing the Kullback-Leibler divergence between the joint probability distributions for both lower-dimensional and higherdimensional data from the input. Since the output from BERTweet has 59,904 dimensions (i.e., 78 tokens in a sequence, each having 768 features given by the hidden units), we first reduced the representation to 100 dimension by applying a Principal Component Analysis (Jolliffe and Cadima, 2016). This way, t-SNE runs faster, without losing too much information when considering the principal components.

The results using the Ensemble-2 configuration are shown in Figure 2 for Tasks 1a, 1b, and 2. Each point represents the embedding of the input sample, while the colors represent the classes or the values for each sample. Based on the ground-truth visualizations (left side), we can observe that the points are not linearly separable. Our best configuration was capable to learn the inner representation of the input data. For the offensive rating task, the examples with higher scores are in the middle of the manifold. We can also observe for the offensive rating task that the majority of classes are biased towards lower rather than higher values.

\section{Conclusions and Future Work}

In this work, we present AMTL-Humor, an adversarial multi-task learning method to deal with the problem of detecting and rating humor and offense for SemEval-2021 Task 7. More specifically, our model is inspired by the ASP-MTL framework and considers BiLSTM and Capsule layers as feature extractors on top of the BERT layer that provides contextualized embeddings.

Two ensemble models were created using variations of our AMTL-Humor architecture. These configurations were trained either on different settings (such as adversarial learning) or by modifying the structure of the branches. We observed that adversarial learning might perform better than other architectures with similar structures, while considering specific configurations and tasks. Another improvement was observed when the model was trained on two tasks using the same branch, namely the AMTL-T1a-Twice model. This approach of duplicating branches may be used in other context as it adds redundancy to the network and supports generalization. Finally, the best results were obtained by using an ensemble over all trained models.

In the future, we aim to study how performance can be improved, especially on Task 1c, by adapting the hierarchical multi-task learning technique (Søgaard and Goldberg, 2016) to the AMTL-Humor architecture. 

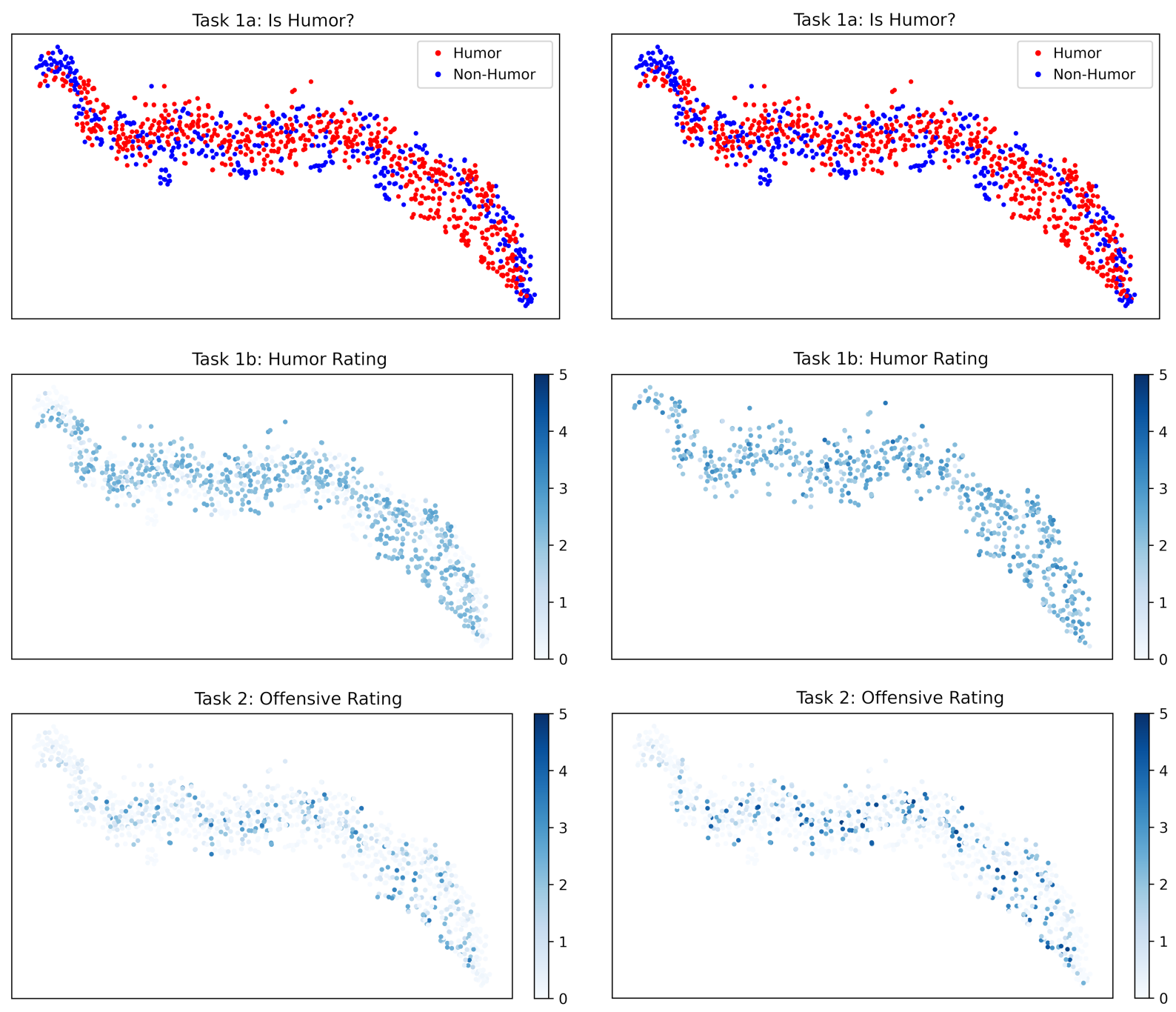

Figure 2: t-SNE projection for the development set data on the output of the BERTweet layer. On left are the plots for predicted outputs, whereas on right are the corresponding plots for ground-truth. Best viewed in color.

\section{References}

Steven Bird, Ewan Klein, and Edward Loper. 2009. Natural Language Processing with Python. O'Reilly Media.

Konstantinos Bousmalis, George Trigeorgis, Nathan Silberman, Dilip Krishnan, and Dumitru Erhan. 2016. Domain separation networks. In Proceedings of the 30th International Conference on Neural Information Processing Systems, pages 343-351.

Costin Busioc, Stefan Ruseti, and Mihai Dascalu. 2020. A literature review of nlp approaches to fake news detection and their applicability to romanianlanguage news analysis. Revista Transilvania, (10).

Kyunghyun Cho, Bart van Merriënboer, Caglar Gulcehre, Dzmitry Bahdanau, Fethi Bougares, Holger Schwenk, and Yoshua Bengio. 2014. Learning phrase representations using rnn encoder-decoder for statistical machine translation. In Proceedings of the 2014 Conference on Empirical Methods in Nat- ural Language Processing (EMNLP), pages 17241734.

Vlad Cristian Dumitru and Traian Rebedea. 2019. Fake and hyper-partisan news identification. In RoCHI, pages $60-67$.

Yaroslav Ganin and Victor Lempitsky. 2015. Unsupervised domain adaptation by backpropagation. In International conference on machine learning, pages 1180-1189. PMLR.

Ian J Goodfellow, Jean Pouget-Abadie, Mehdi Mirza, Bing Xu, David Warde-Farley, Sherjil Ozair, Aaron Courville, and Yoshua Bengio. 2014. Generative adversarial nets. In Proceedings of the 27th International Conference on Neural Information Processing Systems-Volume 2, pages 2672-2680.

Sepp Hochreiter and Jürgen Schmidhuber. 1997. Long short-term memory. Neural computation, 9(8):1735-1780. 
Ian T Jolliffe and Jorge Cadima. 2016. Principal component analysis: a review and recent developments. Philosophical Transactions of the Royal Society A: Mathematical, Physical and Engineering Sciences, 374(2065):20150202.

Diederik P Kingma and Jimmy Ba. 2014. Adam: A method for stochastic optimization. arXiv preprint arXiv:1412.6980.

Kai Lei, Qiuai Fu, and Yuzhi Liang. 2019. Multitask learning with capsule networks. In 2019 International Joint Conference on Neural Networks $(I J C N N)$, pages 1-8. IEEE.

Pengfei Liu, Xipeng Qiu, and Xuan-Jing Huang. 2017. Adversarial multi-task learning for text classification. In Proceedings of the 55th Annual Meeting of the Association for Computational Linguistics (Volume 1: Long Papers), pages 1-10.

Laurens Van der Maaten and Geoffrey Hinton. 2008. Visualizing data using t-sne. Journal of machine learning research, 9(11).

Ana Marasović and Anette Frank. 2018. Srl4orl: Improving opinion role labeling using multi-task learning with semantic role labeling. In Proceedings of the 2018 Conference of the North American Chapter of the Association for Computational Linguistics: Human Language Technologies, Volume 1 (Long Papers), pages 583-594.

J.A. Meaney, Steven R. Wilson, Luis Chiruzzo, Adam Lopez, and Walid Magdy. 2021. Semeval 2021 task 7 , hahackathon, detecting and rating humor and offense. In Proceedings of the 59th Annual Meeting of the Association for Computational Linguistics and the 11th International Joint Conference on Natural Language Processing.

Dat Quoc Nguyen, Thanh Vu, and Anh Tuan Nguyen. 2020. Bertweet: A pre-trained language model for english tweets. In Proceedings of the 2020 Conference on Empirical Methods in Natural Language Processing: System Demonstrations, pages 9-14.

Andrei Paraschiv and Dumitru-Clementin Cercel. 2019. Upb at germeval-2019 task 2: Bert-based offensive language classification of german tweets. In $K O N$ VENS.

Andrei Paraschiv, Dumitru-Clementin Cercel, and Mihai Dascalu. 2020. Upb at semeval-2020 task 11: Propaganda detection with domain-specific trained bert. In Proceedings of the Fourteenth Workshop on Semantic Evaluation, pages 1853-1857.

Kirk Roberts, Michael A Roach, Joseph Johnson, Josh Guthrie, and Sanda Harabagiu. 2012. Empatweet: Annotating and detecting emotions on twitter. In Proceedings of the Eighth International Conference on Language Resources and Evaluation (LREC'12), pages 3806-3813.
Sara Sabour, Nicholas Frosst, and Geoffrey E Hinton. 2017. Dynamic routing between capsules. In Proceedings of the 31st International Conference on Neural Information Processing Systems, pages 3859-3869.

Anders Søgaard and Yoav Goldberg. 2016. Deep multitask learning with low level tasks supervised at lower layers. In Proceedings of the 54th Annual Meeting of the Association for Computational Linguistics (Volume 2: Short Papers), pages 231-235.

Evangelia Spiliopoulou, Eduard Hovy, Alexander G Hauptmann, et al. 2020. Event-related bias removal for real-time disaster events. In Proceedings of the 2020 Conference on Empirical Methods in Natural Language Processing: Findings, pages 3858-3868.

Nitish Srivastava, Geoffrey Hinton, Alex Krizhevsky, Ilya Sutskever, and Ruslan Salakhutdinov. 2014. Dropout: A simple way to prevent neural networks from overfitting. Journal of Machine Learning Research, 15:1929-1958.

Saurabh Srivastava, Puneet Agarwal, Gautam Shroff, Lovekesh Vig, and Vidya Vikas. 2020. Capsule based neural network architecture to perform completeness check for patent eligibility process. In 2020 International Joint Conference on Neural Networks (IJCNN), pages 1-8. IEEE.

Mircea-Adrian Tanase, Dumitru-Clementin Cercel, and Costin Chiru. 2020a. Upb at semeval-2020 task 12: Multilingual offensive language detection on social media by fine-tuning a variety of bert-based models. In Proceedings of the Fourteenth Workshop on Semantic Evaluation, pages 2222-2231.

Mircea-Adrian Tanase, George-Eduard Zaharia, Dumitru-Clementin Cercel, and Mihai Dascalu. 2020b. Detecting aggressiveness in mexican spanish social media content by fine-tuning transformer-based models. In Proceedings of the Iberian Languages Evaluation Forum (IberLEF 2020) co-located with 36th Conference of the Spanish Society for Natural Language Processing (SEPLN) 2020, pages 236-245.

Ashish Vaswani, Noam Shazeer, Niki Parmar, Jakob Uszkoreit, Llion Jones, Aidan N Gomez, Łukasz Kaiser, and Illia Polosukhin. 2017. Attention is all you need. In Proceedings of the 31st International Conference on Neural Information Processing Systems, pages 6000-6010.

George-Alexandru Vlad, Mircea-Adrian Tanase, Cristian Onose, and Dumitru-Clementin Cercel. 2019. Sentence-level propaganda detection in news articles with transfer learning and bert-bilstm-capsule model. In Proceedings of the Second Workshop on Natural Language Processing for Internet Freedom: Censorship, Disinformation, and Propaganda, pages 148-154. 
George-Alexandru Vlad, George-Eduard Zaharia, Dumitru-Clementin Cercel, Costin Chiru, and Stefan Trausan-Matu. 2020a. Upb at semeval-2020 task 8: Joint textual and visual modeling in a multitask learning architecture for memotion analysis. In Proceedings of the Fourteenth Workshop on Semantic Evaluation, pages 1208-1214.

George-Alexandru Vlad, George-Eduard Zaharia, Dumitru-Clementin Cercel, and Mihai Dascalu. 2020b. Upb@ dankmemes: Italian memes analysisemploying visual models and graph convolutional networks for meme identification and hate speech detection. In Proceedings of Seventh Evaluation Campaign of Natural Language Processing and Speech Tools for Italian. Final Workshop (EVALITA 2020), Online. CEUR. org.

Mingxuan Wang. 2019. Towards linear time neural machine translation with capsule networks. In Proceedings of the 2019 Conference on Empirical Methods in Natural Language Processing and the 9th International Joint Conference on Natural Language Processing (EMNLP-IJCNLP), pages 803-812.

Liqiang Xiao, Honglun Zhang, Wenqing Chen, Yongkun Wang, and Yaohui Jin. 2018. Mcapsnet: Capsule network for text with multi-task learning. In Proceedings of the 2018 conference on empirical methods in natural language processing, pages 4565-4574.

Min Yang, Wei Zhao, Jianbo Ye, Zeyang Lei, Zhou Zhao, and Soufei Zhang. 2018. Investigating capsule networks with dynamic routing for text classification. In Proceedings of the 2018 conference on empirical methods in natural language processing, pages 3110-3119.

George-Eduard Zaharia, George-Alexandru Vlad, Dumitru-Clementin Cercel, Traian Rebedea, and Costin Chiru. 2020. Upb at semeval-2020 task 9: Identifying sentiment in code-mixed social media texts using transformers and multi-task learning. In Proceedings of the Fourteenth Workshop on Semantic Evaluation, pages 1322-1330.

Xiabing Zhou, Zhongqing Wang, Shoushan Li, Guodong Zhou, and Min Zhang. 2019. Emotion detection with neural personal discrimination. In Proceedings of the 2019 Conference on Empirical Methods in Natural Language Processing and the 9th International Joint Conference on Natural Language Processing (EMNLP-IJCNLP), pages 5502-5510. 